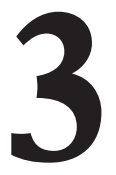

\title{
LA DIMENSIÓN SOCIAL DEL PROCESO DE BOLONIA: APOYOS Y SERVICIOS PARA GRUPOS DE ESTUDIANTES POCO REPRESENTADOS EN LAS UNIVERSIDADES ESPAÑOLAS
}

\author{
(SOCIAL DIMENSION OF THE BOLOGNA PROCESS: SUPPORT \\ AND SERVICES FOR UNDER-REPRESENTED GROUPS IN SPANISH \\ UNIVERSITIES)
}

Inmaculada Egido Gálvez y M. ${ }^{a}$ José Fernández Díaz Universidad Complutense de Madrid

Arturo Galán

Universidad Nacional de Educación a Distancia

DOI: 10.5944/educxx1.17.2.11479

\section{Cómo referenciar este artículo/How to reference this article:}

Egido Gálvez, I.; Fernández Díaz, M. J. y Galán, A. (2014). La dimensión social del proceso de Bolonia: Apoyos y servicios para grupos de estudiantes poco representados en las universidades españolas. Educación XX1, 17 (2), 57-81. doi: 10.5944/educxx1.17.2.11479

Egido Gálvez, I.; Fernández Díaz, M. J. y Galán, A. (2014). Social dimension of the Bologna Process: Support and services for under-represented groups in Spanish universities. Educación XX1, 17 (2), 57-81. doi: 10.5944/educxx1.17.2.11479

\section{RESUMEN}

La dimensión social del Espacio Europeo de Educación Superior (EEES) es una de las facetas menos conocidas del proceso de Bolonia, a pesar de que constituye un elemento clave para el logro de la igualdad de oportunidades en la enseñanza superior. En concreto, dicha dimensión implica la necesidad de que todos los colectivos de estudiantes, independientemente de sus circunstancias personales o sociales, tengan la oportunidad de acceder a la universidad y terminar con éxito sus estudios. En este artículo, tras un breve repaso al concepto de dimensión social, se revisa la situación de las universidades españolas en relación a tres colectivos con escasa presencia en la enseñanza universitaria: adultos, inmigrantes y personas con discapacidad. La conclusión general del trabajo es que resulta necesario seguir ampliando los apoyos para estos estudiantes, intensificando el esfuerzo realizado a este respecto en los pasados años.

\section{PALABRAS CLAVE}

Educación universitaria, Espacio Europeo de Educación Superior, Servicios de apoyo a estudiantes, España. 


\section{ABSTRACT}

The social dimension of EHEA (European Higher Education Area) is one of the less known aspects of the Bologna Process, although it is a key element for equality of opportunities in the higher education level. This dimension implies that all students, irrespective of their personal or social circumstances, have the same opportunity to access and successfully finish their studies. This article, after a brief analysis of the social dimension concept, reviews the situation of Spanish universities for three under-represented groups: adults, immigrants and people with disabilities. The main conclusion is that it necessary to continue increasing support for these students, increasing the efforts made in this respect in recent years.

\section{KEYWORDS}

Higher Education, European Higher Education Area, Student, Academic Support Services, Spain

\section{INTRODUCCIÓN}

Hace ya más de una década que comenzó el proceso de creación del Espacio Europeo de Educación Superior (EEES). Dicho proceso se ha materializado en los países participantes en la implantación de un conjunto de reformas dirigidas a incrementar la movilidad de estudiantes y profesores y a facilitar el reconocimiento de los periodos de estudio y de las titulaciones universitarias. Sin embargo, aunque esas líneas de convergencia sean las más conocidas, lo cierto es que el EEES responde también a otras finalidades de más amplio alcance, como contribuir a la creación de un sistema de enseñanza superior del que todas las personas puedan tomar parte en condiciones de igualdad (European Commission, 2001 y 2005; EUA, 2005).

En línea con esta finalidad, uno de los retos a los que se enfrenta el EEES en la actualidad es el de hacer la enseñanza superior igualmente accesible para el conjunto de los ciudadanos, creando las condiciones necesarias para que todos puedan completar sus estudios sin encontrar obstáculos relacionados con su bagaje social, personal o económico. Este reto, que constituye la dimensión social del proceso de Bolonia, implica que las universidades deben abrirse a públicos nuevos y más amplios que en el pasado (BFUG, 2007). Se requiere, por tanto, la creación de un sistema capaz de acoger a todo tipo de estudiantes, especialmente a aquellos que, por diversas razones, tienen más dificultades para culminar con éxito los estudios universitarios. 
En este artículo, tras realizar un breve repaso a la idea de la dimensión social del proceso de Bolonia y a su evolución a lo largo de los pasados años, se profundiza en los servicios y apoyos disponibles en las universidades españolas para tres de los colectivos de estudiantes que encuentran mayores obstáculos para cursar estudios universitarios. En concreto, los colectivos que se analizan son los estudiantes con discapacidad, los adultos y los inmigrantes. Aunque, sin duda, con esta revisión únicamente se esboza una primera aproximación a este tema, que requiere la realización de estudios que lo aborden con mayor exhaustividad, el trabajo permite obtener una visión general sobre el estado actual de nuestras universidades en relación con los servicios dirigidos a algunos de los estudiantes que presentan mayores dificultades y, por ende, en relación a la dimensión social del proceso de Bolonia.

\section{LA TRANSFORMACIÓN DE LA UNIVERSIDAD EUROPEA Y LA «DIMENSIÓN SOCIAL» DEL PROCESO DE BOLONIA}

En los primeros pasos de la construcción del EEES el principio de igualdad de oportunidades en la enseñanza universitaria fue objeto de una escasa atención, al menos por lo que se refiere a su plasmación explícita en los documentos de carácter oficial. Así, tanto la Declaración de la Sorbona (1998) como la de Bolonia (1999) omitieron esta idea, centrándose en las reformas relacionadas con la estructura de las titulaciones, el reconocimiento de estudios, la movilidad o la competitividad del área europea de educación superior. No obstante, poco tiempo después, en la Conferencia de Praga celebrada en el año 2001, los ministros de los países participantes en el EEES hicieron referencia, a instancias de los estudiantes, a la dimensión social del proceso de Bolonia (Eurostat-Eurostudent, 2009). Aunque en esa Conferencia la dimensión social se trató como un aspecto vinculado casi exclusivamente a las posibilidades de movilidad de los estudiantes, lo cierto es que ya en ese momento se planteó que una de las principales metas del proceso de convergencia era la de crear un sistema de enseñanza superior basado en el principio de aprendizaje permanente, del que todas las personas, sin otros condicionantes que los derivados de sus propias capacidades, tuvieran la posibilidad de formar parte (Comunicado de la Conferencia de Ministros Europeos responsables de Educación Superior, 2001).

En la siguiente reunión de seguimiento del proceso de Bolonia, que tuvo lugar en Berlín en el año 2003, hubo ya una alusión más precisa a esta temática, puesto que en ella los ministros plantearon la importancia de buscar un equilibrio entre el incremento de la competitividad del EEES y la necesidad de mejorar sus características sociales. La Declaración resultante de esta conferencia manifiesta así el compromiso explícito de los 
participantes con la idea de hacer que la educación superior sea igualmente accesible para todos, en función de sus capacidades, utilizando los medios necesarios al efecto. Destaca también la importancia de que los estudiantes cuenten con condiciones adecuadas para estudiar y para vivir, de manera que puedan terminar sus estudios en un plazo apropiado, sin encontrar trabas en su entorno (Comunicado de la Conferencia de Ministros Europeos responsables de Educación Superior, 2003).

Avanzando en esa dirección, la Conferencia de Ministros de Bergen (2005) estableció formalmente que la dimensión social del Proceso de Bolonia forma parte del EEES. De hecho, en el Comunicado de esta Conferencia se dedicó un epígrafe completo a la dimensión social como un aspecto crucial para el éxito del proceso de Bolonia. En palabras de los ministros:

«La dimensión social del Proceso de Bolonia es parte integrante del EEES y una condición necesaria para el atractivo y la competitividad del EEES. [...] La dimensión social incluye medidas adoptadas por los gobiernos para ayudar a los estudiantes, especialmente a los sectores socialmente desfavorecidos, en aspectos financieros y económicos y proporcionarles servicios de orientación y asesoramiento con vistas a facilitar un acceso más amplio.» (Comunicado de la Conferencia de Ministros Europeos responsables de Educación Superior, 2005, p. 4).

Las Conferencias de Ministros posteriores, que tuvieron lugar en Londres (2007), Lovaina (2009) y Bucarest (2012), reiteraron el compromiso con la dimensión social del proceso de convergencia. Así, en Londres se destacó el papel esencial que la enseñanza superior debe jugar en la promoción de la cohesión social, para lo que resulta necesario facilitar servicios adecuados a los estudiantes, crear itinerarios de aprendizaje más flexibles y ampliar la participación (Comunicado de la Conferencia de Ministros Europeos responsables de Educación Superior, 2007). En Lovaina el acceso equitativo a la Universidad volvió a señalarse como uno de los retos del proceso de convergencia, indicando textualmente que:

"El alumnado de educación superior debe reflejar la diversidad de poblaciones de Europa. Por tanto, [...] el acceso a la educación superior debe ampliarse fomentando el potencial de los alumnos de grupos infrarrepresentados y proporcionándoles las condiciones adecuadas para que puedan terminar sus estudios.» (Comunicado de la Conferencia de Ministros Europeos responsables de Educación Superior, 2009, p. 9).

Finalmente, la Conferencia de Bucarest puso el énfasis en la necesidad de que todos los países adopten medidas destinadas a ampliar el acceso a una educación superior de calidad, reforzando las estrategias acordadas 
en Londres e incluyendo entre ellas el reconocimiento de los aprendizajes previos de los estudiantes (Comunicado de la Conferencia de Ministros Europeos responsables de Educación Superior, 2012).

Pero no solo las Conferencias de los Ministros han tratado la dimensión social del EEES, sino que tanto las instituciones universitarias que forman parte de la Asociación de la Universidad Europea (EUA: European University Association) como los sindicatos de estudiantes, representados por medio de la Unión de Estudiantes Europeos (ESU: European Students' Union), han impulsado esta idea, reivindicando la necesidad de desarrollar políticas para ampliar las oportunidades de acceso y apoyo a todos los grupos de estudiantes, así como la importancia de fomentar la investigación en esta materia, con el fin de evitar la discriminación en los sistemas de educación superior (EUA, 2005; ESIB, 2003 y 2007; ESU, 2012).

Recopilando lo anterior, puede afirmarse que la dimensión social es una parte esencial del proceso de convergencia universitaria europeo. Si bien podría argumentarse que los documentos mencionados son primordialmente una exposición de intenciones, circunscritas al plano de las declaraciones, lo cierto es que existe un mecanismo de seguimiento destinado a controlar los progresos de los países participantes en este terreno. Así, el Grupo de Seguimiento del Proceso de Bolonia (BFUG) cuenta con un equipo de trabajo dedicado a Dimensión Social y Movilidad. Dicho grupo ha tenido, entre otras funciones, un papel muy importante en la construcción del propio concepto de «dimensión social» y está centrado en estos momentos en la recopilación de datos comparables sobre la situación social y económica de los estudiantes en los países integrantes del EEES (Eurydice, 2011).

En relación al concepto de dimensión social, el Grupo de Seguimiento ha adoptado un enfoque amplio, que permite dar cabida a la diversidad entre los países participantes (BFUG, 2007). De este modo, bajo el epígrafe de dimensión social se incluyen todas las acciones encaminadas a la meta de alcanzar la igualdad de oportunidades en términos de acceso, participación y éxito en los estudios para toda la población. En concreto, el Consejo de la Unión Europea define la dimensión social como:

"La igualdad de oportunidades para acceder a una educación de calidad, así como la equidad en el tratamiento, incluyendo servicios adaptados a las necesidades individuales» (Council of European Union, 2010, p. 2).

Por lo que se refiere a su seguimiento, existe una voluntad decidida de avanzar en su logro, de manera que todos los países han emprendido un Plan de Acción en relación con la dimensión social (BFUG, 2007; Eurostat- 
Eurostudent, 2009). El primer paso de ese Plan es identificar los grupos de población que se encuentran poco representados en la enseñanza superior y, de acuerdo con las conclusiones de la Conferencia de Lovaina, elaborar los indicadores destinados al seguimiento de los progresos relacionados con esta dimensión. Entre los estudiantes que tienen mayores dificultades para tomar parte en la enseñanza universitaria se incluyen todos aquellos que por sus particulares circunstancias individuales, sociales o familiares tienen una representación menor entre el alumnado universitario que el resto de los grupos. Se trata de las personas con algún tipo de discapacidad, de quienes proceden de los estratos socioeconómicos más bajos, de los inmigrantes, de las personas con cargas familiares, de quienes trabajan y de otros colectivos que tradicionalmente no han formado parte del alumnado "convencional» de las universidades (Comunicado de la Conferencia de Ministros Europeos Responsables de Educación Superior, 2007).

\section{MÉTODO}

\section{Unidad de análisis}

En el presente estudio, se han utilizado los documentos relacionados con la dimensión social del EEES. Para ello, se han revisado los documentos emanados por las conferencias de ministros, por la Comisión Europea, los grupos profesionales, asociaciones y sindicatos de estudiantes de nivel europeo relacionados con grupos de estudiantes con dificultades de acceso a la Educación Superior y, finalmente, documentos legislativos a nivel nacional con influencia en este ámbito de estudio.

Además, se ha realizado una revisión explícita de los proyectos de investigación financiados que han abordado esta temática en los últimos años, con el fin de reflejar la evolución de los problemas objeto de estudio y los avances logrados.

\section{Diseño y procedimiento}

El presente trabajo se clasifica como estudio descriptivo mediante análisis de documentos (Montero y León, 2007) y se ha redactado según las normas establecidas por Ramos-Álvarez, Moreno-Fernández, Valdés-Conroy y Catena (2008).

Después de una minuciosa búsqueda de bibliografía al respecto, los autores del presente trabajo han realizado un análisis exhaustivo del contenido de las páginas Web de las universidades españolas. Con el fin de 
sistematizar la recogida de información y garantizar en la medida de lo posible la objetividad del análisis, se diseñó un instrumento de recogida de datos adaptado al objetivo concreto de este estudio. Dicho instrumento fue elaborado siguiendo los principios generales de la metodología de análisis de contenido como procedimiento destinado a estudiar de manera rigurosa los contenidos de las comunicaciones (McMillan y Schumacher, 2005), si bien en este caso concreto el análisis se centró básicamente en rasgos de carácter cualitativo, como la presencia o ausencia de los servicios de apoyo en la información aportada por las páginas Web. El punto de partida para la elaboración del instrumento fue precisar las categorías de análisis de los apoyos que pueden ofrecer las Universidades a través de los servicios correspondientes en cada caso. En este sentido, se consideró como primer elemento de categorización los tres grupos de alumnos con distintos tipos de necesidades: estudiantes inmigrantes, adultos y con discapacidad. Tras la definición precisa de estos tipos, se concretaron tres momentos dentro de cada una de las categorías anteriores de análisis, que responden a tres periodos diferentes de la trayectoria educativa de los estudiantes en la Universidad: acceso a los estudios, desarrollo de la carrera e inserción laboral.

La definición de estos tres momentos en la trayectoria universitaria de los colectivos estudiados parte del concepto clásico de igualdad de oportunidades educativas (Delors, 1996) y es la que contempla la Red Europea de expertos sobre Planificación de políticas para implementar la educación inclusiva (Mapping the Implementation of Policy for Inclusive Education, MIPIE). Dicha Red recomienda precisamente esa estructura con el fin de crear un marco común para la recogida de datos sobre la educación inclusiva tanto a nivel nacional como internacional (European Agency for Development in Special Needs Education, 2011). En ella se considera la triple acepción del concepto de educación inclusiva: escolarización (acceso a la educación y a las instituciones escolares), participación (calidad de las experiencias de aprendizaje) y logros (resultados).

Una vez definidas estas categorías generales, se especificaron los tipos de apoyo concretos que fueron utilizados como unidades de análisis. Así, en relación con el acceso a los estudios se contempla la existencia de servicios de información y apoyo para el ingreso en la universidad y las ayudas económicas para la matrícula. En relación con el desarrollo de la carrera se examina el conjunto de posibles ayudas formativas-académicas (servicios de orientación y apoyo académico), extra-académicas (actividades artísticas, culturales o deportivas) y apoyos económicos para el desarrollo de la carrera (alojamiento, transporte, manutención o ayudas a la movilidad). Por último, en relación con la inserción laboral se consideran los apoyos para la transición a la vida activa y las posibilidades de formación permanente. 


\section{RESULTADOS}

Se presentan los resultados agrupados en dos grandes apartados. Por una parte, la situación de los grupos de estudiantes que pueden encontrarse en desventaja frente a los estudiantes considerados normales o estudiantes prototipo. En un segundo epígrafe se estudia la respuesta dada a aquellos estudiantes a través de distintos programas y servicios de apoyo específicos ofrecidos por las instituciones.

\section{Los grupos de estudiantes poco representados en las universidades españolas}

Como se ha mencionado, un primer paso a la hora de profundizar en la dimensión social del sistema universitario es identificar a los colectivos de estudiantes con escasa representación en la educación superior, o, lo que es igual, a aquellos grupos que pueden encontrarse en situación de desventaja respecto a los demás, puesto que dicha identificación constituye un requisito previo necesario para poder diseñar los servicios y apoyos específicos destinados a estos estudiantes.

A este respecto, la bibliografía publicada en España permite constatar un importante progreso desde la incorporación de nuestro país al proceso de Bolonia hasta este momento. Así, una revisión de la información disponible sobre las características de los estudiantes universitarios y sobre la representación de los diferentes colectivos en la enseñanza superior desde el comienzo de la década de los 2000 hasta la actualidad pone de manifiesto cómo ésta se ha ampliado de manera notable.

Concretamente, en el inicio de la década de los 2000, los datos sobre los estudiantes universitarios en España procedían casi exclusivamente del Ministerio de Educación, que a través de diversos Informes sobre el Sistema Educativo Español ${ }^{1}$ y de las Estadísticas de Estudiantes Universitarios ${ }^{2}$ proporcionaba algunas referencias sobre esta cuestión. También el Instituto Nacional de Estadística (INE) publicaba anualmente las Estadísticas sobre la Enseñanza Universitaria en España, en las que se incluían informaciones sobre los estudiantes del nivel superior. Estas fuentes, sin embargo, ofrecían mayoritariamente datos de carácter global, por lo que existía una carencia de estudios que abordaran específicamente las desigualdades de participación en la enseñanza universitaria.

Esta escasez de datos empezó a solventarse al incorporarse España al proyecto Eurostudent, destinado a observar con detalle las características socioeconómicas de los estudiantes universitarios, de acuerdo con indicado- 
res comparables entre países. En concreto, España se incorporó a este proyecto en el año 2005 y ha participado en dos ediciones sucesivas del mismo, lo que ha permitido disponer no sólo de indicadores estadísticos sobre las condiciones de vida y las características de los universitarios españoles, sino también de un buen número de análisis al respecto (Ariño y Llopis, 2011a; Fernández Fernández, 2008; Ruesga 2006 y 2008; Ruesga y Heredero, 2008). Junto a estos estudios, otros informes, realizados desde organizaciones de carácter público y privado, han enriquecido en gran medida la información disponible sobre las tipologías de estudiantes en nuestro sistema de educación superior. Así, los trabajos realizados por la Conferencia de Rectores de las Universidades Españolas (CRUE) (Hernández Armenteros, 2002, 2004, 2006 y 2008; Michavila, 2012) y las Encuestas sobre los estudiantes universitarios españoles realizadas por la Fundación BBVA (Fundación BBVA, 2003, 2005, 2006 y 2010) aportan también valiosas informaciones sobre este tema.

Los estudios citados, que abordan el conjunto de la población estudiantil, han venido a completarse con otros dedicados específicamente a algunos colectivos concretos. Así, en relación con la presencia y situación de los estudiantes con discapacidad en las universidades el progreso también ha sido enorme (entre otros, Alcedo et al., 2007; Forteza y Ortego, 2003; CERMI, 2004; González-Badía y Molina, 2006; Real Patronato sobre Discapacidad, 2007). En el caso de los estudiantes inmigrantes, aunque el número de estudios no ha sido tan amplio, también contamos en este momento con una información relativamente completa (Cachón, 2003; INJUVE, 2008; Lebrero y Quicios, 2010; Pena y Galindo, 2005).

En la actualidad España cuenta ya con un Observatorio de la vida y participación de los estudiantes similar a los que pueden encontrarse en otros países europeos. Este Observatorio presentó en el año 2011 la Encuesta sobre Condiciones de vida y participación de los estudiantes universitarios en España (Ariño y Llopis, 2011b) en la que participaron más de 45.000 estudiantes y en la que se recogen con detalle informaciones sobre los perfiles de los estudiantes, así como sobre sus condiciones académicas y sociales. Existe también el Observatorio Universidad y Discapacidad, promovido por la fundación ONCE y la Universidad Politécnica de Cataluña, con el fin de elaborar estudios sobre la situación de los estudiantes con discapacidad en las universidades españolas.

El progreso experimentado en la recopilación de datos durante estos últimos años permite disponer actualmente de un conjunto de informaciones relativamente amplias para detectar la posible existencia de grupos o colectivos de estudiantes poco representados en nuestra enseñanza superior, en línea con la definición de la dimensión social del proceso de Bolonia comentada anteriormente. En concreto, una síntesis de los indicadores que 
figuran en los estudios citados permite afirmar que España ha avanzado en los últimos años hacia la construcción de una educación superior más equitativa, especialmente en lo que se refiere al acceso de los distintos sectores sociales a la universidad, aunque todavía pueden encontrarse diferencias importantes en las tasas de participación en la enseñanza universitaria en función del nivel cultural de las familias (Ariño y Llopis, 2011a; Orr, Gwosc y Netz, 2011). Estos estudios indican también que, en comparación con otros países, en España siguen siendo relativamente pocos los estudiantes de edades medias o maduras, por lo que se considera necesario que nuestras universidades ofrezcan vías alternativas para aquellas personas que, por la razón que fuere, han tenido que interrumpir sus estudios o no pudieron acceder a ellos antes de los 25 años (Ariño y Llopis, 2011b).

Además de lo anterior, ciertas variables parecen interferir todavía de manera negativa en el acceso a la enseñanza universitaria. Así, entre otros grupos, siguen siendo pocos los inmigrantes tanto de primera como de segunda generación que acceden a la universidad, ya que estos solo representan el 2,3\% de la población estudiantil, cuando su presencia en el conjunto de la sociedad es superior al 11\% (Secretaría General de Universidades, 2011). De igual modo, y pesar de los avances de los últimos años, se constata que los estudiantes con discapacidad solo representan el 0,5\% del alumnado universitario, cuando el porcentaje de jóvenes con discapacidad se encuentra entre el $2 \%$ y el $2,5 \%$ en la sociedad (Real Patronato sobre Discapacidad, 2007).

\section{Programas y servicios de apoyo a colectivos de estudiantes en situación de desventaja en las Universidades españolas}

Una vez identificados los grupos menos representados en las universidades españolas, una cuestión central a la dimensión social de la enseñanza superior es la existencia de apoyos específicos para dichos colectivos, con el fin de proporcionarles las condiciones adecuadas para que puedan cursar con éxito sus estudios. Por tanto, resulta pertinente plantearse qué tipo de medidas existen en las universidades españolas para que los estudiantes con más dificultades se integren en ellas, así como hasta qué punto dichas medidas pueden considerarse generalizadas y cumplen adecuadamente con sus objetivos. Para realizar una aproximación a esta cuestión en este trabajo se ha seguido una doble metodología. En primer lugar, se ha realizado una búsqueda de los programas y servicios ofertados a estos estudiantes en las páginas Web de las universidades españolas. En segundo lugar, se ha llevado a cabo la revisión de un conjunto de investigaciones realizadas sobre esta temática, para profundizar en mayor medida en el funcionamiento de dichos servicios. Por supuesto, esta doble vía aporta sólo una primera aproxima- 
ción al conocimiento de las medidas existentes en las universidades españolas para los estudiantes con escasa representación, aproximación que debe completarse con investigaciones adicionales orientadas a profundizar en esta cuestión.

Dada la imposibilidad de abordar en este artículo todos los grupos de estudiantes en situación de desventaja en el sistema universitario español, se tratan específicamente tres de ellos: los estudiantes de origen inmigrante, los estudiantes adultos y los estudiantes con discapacidad. Evidentemente, entre estos colectivos existen claras diferencias tanto de índole cuantitativa como cualitativa. No obstante, los tres grupos han sido identificados de acuerdo con los indicadores estadísticos comentados anteriormente como colectivos escasamente presentes en nuestro sistema de enseñanza superior, por lo que precisan de apoyos y programas destinados a garantizar no sólo su acceso a la universidad, sino la posibilidad de culminar sus estudios.

Servicios ofertados por las universidades españolas para los estudiantes de origen inmigrante, adultos y con discapacidad

La revisión de las páginas Web de las universidades españolas permite obtener una visión de conjunto sobre los servicios ofertados en las mismas para los tres colectivos indicados. Por ello se ha consultado la información disponible en Internet en un total de 75 universidades, tanto públicas como privadas y tanto presenciales como a distancia.

Los resultados de dicha consulta de acuerdo con las categorías utilizadas aparecen sintetizados en la tabla 1, que ofrece, a modo de panorámica, una síntesis de los principales apoyos destinados de manera específica a estos estudiantes, aún cuando es necesario tener en cuenta que muchos de los servicios de carácter general que las universidades ofrecen al conjunto de sus alumnos pueden ser utilizados también por estos grupos.

Como era lógico esperar, la información de las Webs permite constatar la existencia de una amplia variabilidad en las universidades españolas en cuanto a los servicios destinados a los grupos de estudiantes definidos. Dicha variabilidad se encuentra no sólo en los grados de desarrollo de los servicios disponibles, sino también en la manera de ofertarlos y en los organismos responsables de los mismos. A este respecto, y aunque la finalidad de este artículo no es mencionar universidades concretas, debe señalarse que la Universidad Nacional de Educación a Distancia (UNED) es la institución que destaca más claramente en la oferta de servicios dirigidos a los tres colectivos de estudiantes señalados. 
Además de lo anterior, cabe afirmar que los servicios destinados a los estudiantes adultos y, sobre todo, a los inmigrantes, presentan una implantación muy desigual en las distintas universidades españolas, lo que permite suponer que la respuesta institucional para estos alumnos es muy diferente en unos casos y en otros. En relación a los estudiantes con discapacidad, aunque también se aprecian diferencias entre universidades, estas no son tan acusadas y cuentan con un desarrollo más amplio en la mayoría de ellas. Como puede apreciarse en la tabla, para los estudiantes con discapacidad existen apoyos en los distintos momentos de la trayectoria académica de los estudiantes. Por el contrario, los servicios ofertados para los estudiantes de origen inmigrante y para los adultos se centran casi exclusivamente en el momento de acceso a la universidad, sin que apenas se hayan desarrollado apoyos destinados a facilitar la realización con éxito de los estudios de estos colectivos o su integración en el mundo laboral, una vez finalizada la carrera.

\begin{tabular}{|c|c|c|}
\hline $\begin{array}{c}\text { Colectivo } \\
\text { de estudiantes }\end{array}$ & $\begin{array}{l}\text { Momento } \\
\text { de la trayectoria } \\
\text { del estudiante }\end{array}$ & Apoyos existentes \\
\hline \multirow[t]{3}{*}{$\begin{array}{l}\text { Estudiantes } \\
\text { de origen } \\
\text { inmigrante }\end{array}$} & Acceso a los estudios & $\begin{array}{l}\text { - Becas y ayudas económicas especí- } \\
\text { ficas para inmigrantes } \\
\text { - Cursos de pre-acceso para extran- } \\
\text { jeros } \\
\text { - Oficinas de información y orienta- } \\
\text { ción a inmigrantes } \\
\text { - Guías de acceso para estudiantes } \\
\text { extranjeros } \\
\text { - Pruebas de acceso para extranje- } \\
\text { ros } \\
\text { - Asesoría legal } \\
\text { - Servicios de traducción } \\
\text { - Ayudas para la convalidación y } \\
\text { homologación de estudios } \\
\text { - Cursos de lengua y cultura espa- } \\
\text { ñola }\end{array}$ \\
\hline & Desarrollo de la carrera & \\
\hline & Inserción laboral & \\
\hline \multirow{3}{*}{$\begin{array}{l}\text { Estudiantes } \\
\text { adultos }\end{array}$} & Acceso a los estudios & $\begin{array}{l}\text { - Acceso para mayores de } 25,40 \text { y } \\
45 \text { años } \\
\text { - Reconocimiento de la experien- } \\
\text { cia laboral y de la formación ex- } \\
\text { trauniversitaria }\end{array}$ \\
\hline & Desarrollo de la carrera & $\begin{array}{l}\text { - Servicios y ayudas para estudian- } \\
\text { tes con hijos: guarderías, escuelas } \\
\text { infantiles, campamentos, etc. }\end{array}$ \\
\hline & Inserción laboral & \\
\hline
\end{tabular}




\begin{tabular}{|c|c|c|}
\hline $\begin{array}{c}\text { Colectivo } \\
\text { de estudiantes }\end{array}$ & $\begin{array}{l}\text { Momento } \\
\text { de la trayectoria } \\
\text { del estudiante }\end{array}$ & Apoyos existentes \\
\hline \multirow{3}{*}{$\begin{array}{l}\text { Estudiantes con } \\
\text { discapacidad }\end{array}$} & Acceso a los estudios & $\begin{array}{l}\text { - Reserva de cupos de matrícula } \\
\text { - Exención total o parcial de tasas } \\
\text { - Eliminación de barreras arquitec- } \\
\text { tónicas: rampas de acceso, ascen- } \\
\text { sores, mobiliario ergonómico, etc. } \\
\text { - Oficinas de información para es- } \\
\text { tudiantes con discapacidad }\end{array}$ \\
\hline & Desarrollo de la carrera & $\begin{array}{l}\text { - Servicios de transporte específicos } \\
\text { - Alojamientos adaptados } \\
\text { - Programas de acompañamiento } \\
\text { - Accesibilidad de Webs y docu- } \\
\text { mentos electrónicos } \\
\text { - Servicios de atención a alumnos } \\
\text { con discapacidad } \\
\text { - Servicios de orientación, asesora- } \\
\text { miento y apoyo académico espe- } \\
\text { cializado } \\
\text { - Adaptación de pruebas y materia- } \\
\text { les de enseñanza } \\
\text { - Campañas de sensibilización de la } \\
\text { comunidad universitaria } \\
\text { - Protocolos para atención a estu- } \\
\text { diantes con discapacidad } \\
\text { - Programas de diagnóstico y detec- } \\
\text { ción de necesidades } \\
\text { - Tutorías y redes de mentoría } \\
\text { - Adaptaciones curriculares } \\
\text { - Servicios de deporte adaptado } \\
\text { - Asistencia médica específica }\end{array}$ \\
\hline & Inserción laboral & $\begin{array}{l}\text { - Servicios de orientación e inser- } \\
\text { ción laboral para estudiantes con } \\
\text { discapacidad }\end{array}$ \\
\hline
\end{tabular}

Fuente: Elaboración propia a partir de la revisión de las páginas Web de las universidades españolas

Tabla 1. Apoyos existentes en las universidades españolas para los estudiantes de origen inmigrantes, adultos y con discapacidad 
Revisión de investigaciones sobre programas y servicios de apoyo a estudiantes

Con el fin de profundizar en la información obtenida a través las páginas Web de las universidades, se ha realizado una revisión de investigaciones sobre el funcionamiento de los servicios destinados a los tres colectivos de estudiantes definidos. Concretamente, se han analizado las investigaciones dedicadas a esta temática dentro del Programa Estudios y Análisis de la Dirección General de Universidades del Ministerio de Educación. Este programa, que lleva ya más de una década de funcionamiento, ha perfilado en sus sucesivas convocatorias varias líneas de investigación relacionadas con la dimensión social de la enseñanza universitaria y con los programas y servicios de apoyo a los estudiantes. Como ya se ha mencionado, la revisión realizada no pretende, en ningún caso, ofrecer una panorámica de la totalidad de la investigación que a este respecto existe en nuestro país, sino únicamente proporcionar una primera aproximación al funcionamiento de los servicios destinados a estos colectivos de estudiantes en nuestras universidades.

Como en el caso anterior, el instrumento de recogida y análisis de la información se ha elaborado ad hoc para este estudio, estableciendo como categorías de análisis el año de realización de la investigación, el ámbito geográfico considerado, los colectivos de estudiantes analizados y las principales conclusiones de los estudios. Los servicios y tipos de apoyo objeto de los distintos estudios se ha categorizado de la misma manera que en el caso de las páginas Web.

En la Tabla 2 se presentan ordenadas cronológicamente las investigaciones financiadas por el programa Estudios y Análisis que han sido objeto de revisión. En conjunto, se ha trabajado con un total de 21 investigaciones. Se han excluido aquellos estudios que tratan específicamente las Universidades de Mayores, por considerar que la naturaleza de esos programas es distinta de la de los estudios reglados ofertados para el conjunto de estudiantes.

\begin{tabular}{|l|c|l|l|}
\hline Director & Año & \multicolumn{1}{|c|}{ Título de la investigación } & \multicolumn{1}{|c|}{$\begin{array}{c}\text { Colectivo } \\
\text { considerado }\end{array}$} \\
\hline Llorent & 2003 & $\begin{array}{l}\text { Servicios al estudiante en las Universidades } \\
\text { españolas. Estudio comparado }\end{array}$ & $\begin{array}{l}\text { Inmigrantes } \\
\text { Adultos } \\
\text { Con discapa- } \\
\text { cidad }\end{array}$ \\
\hline Castellana & 2005 & $\begin{array}{l}\text { Estudio sobre la atención a la diversidad } \\
\text { dentro de las aulas universitarias }\end{array}$ & $\begin{array}{l}\text { Con discapa- } \\
\text { cidad }\end{array}$ \\
\hline
\end{tabular}




\begin{tabular}{|c|c|c|c|}
\hline Director & Año & Título de la investigación & $\begin{array}{l}\text { Colectivo } \\
\text { considerado }\end{array}$ \\
\hline Alfaro & 2006 & $\begin{array}{l}\text { La respuesta organizativa y metodológica de } \\
\text { la universidad ante una nueva tipología de } \\
\text { estudiantes }\end{array}$ & $\begin{array}{l}\text { Con discapa- } \\
\text { cidad }\end{array}$ \\
\hline $\begin{array}{l}\text { Fernández } \\
\text { Díaz }\end{array}$ & 2006 & $\begin{array}{l}\text { Necesidades de los estudiantes universitarios } \\
\text { ante la realidad del EEES }\end{array}$ & $\begin{array}{l}\text { Inmigrantes } \\
\text { Adultos } \\
\text { Con discapa- } \\
\text { cidad }\end{array}$ \\
\hline Llorent & 2006 & $\begin{array}{l}\text { Valoración de los estudiantes universitarios } \\
\text { sobre los servicios ofertados en sus respecti- } \\
\text { vas Universidades. Análisis y estudio compa- } \\
\text { rado }\end{array}$ & $\begin{array}{l}\text { Con discapa- } \\
\text { cidad }\end{array}$ \\
\hline $\begin{array}{l}\text { Díez } \\
\text { Villoria }\end{array}$ & 2007 & $\begin{array}{l}\text { Necesidad, predisposición y ajuste para el } \\
\text { uso de tecnologías de ayuda en estudiantes } \\
\text { universitarios con discapacidad: adaptación } \\
\text { y validación de un instrumento de evaluación }\end{array}$ & $\begin{array}{l}\text { Con discapa- } \\
\text { cidad }\end{array}$ \\
\hline Mirón & 2007 & $\begin{array}{l}\text { Proyecto para la mejora de la calidad total de } \\
\text { la enseñanza superior en relación con alum- } \\
\underline{\text { nos con discapacidad }}\end{array}$ & $\begin{array}{l}\text { Con discapa- } \\
\text { cidad }\end{array}$ \\
\hline Alba & 2008 & $\begin{array}{l}\text { Servicios de apoyo tecnológico y didáctico } \\
\text { para mejorar la accesibilidad de la enseñan- } \\
\text { za universitaria para las personas con disca- } \\
\text { pacidad }\end{array}$ & $\begin{array}{l}\text { Con discapa- } \\
\text { cidad }\end{array}$ \\
\hline Guasch & 2008 & $\begin{array}{l}\text { Guía de actividades docentes para la forma- } \\
\text { ción en integración e igualdad de oportuni- } \\
\text { dades por razón de discapacidad en las ense- } \\
\text { ñanzas técnicas }\end{array}$ & $\begin{array}{l}\text { Con discapa- } \\
\text { cidad }\end{array}$ \\
\hline Herrera & 2008 & $\begin{array}{l}\text { Estudio para la implantación de un sistema } \\
\text { de tutorías virtuales destinado a alumnos } \\
\text { con discapacidad en el entorno universitario }\end{array}$ & $\begin{array}{l}\text { Con discapa- } \\
\text { cidad }\end{array}$ \\
\hline $\begin{array}{l}\text { Pérez } \\
\text { Serrano }\end{array}$ & 2008 & $\begin{array}{l}\text { Hacia una universidad inclusiva. Medidas de } \\
\text { apoyo al alumnado inmigrante }\end{array}$ & Inmigrantes \\
\hline $\begin{array}{l}\text { Álvarez } \\
\text { Pérez }\end{array}$ & 2009 & $\begin{array}{l}\text { Programa de tutoría de carrera para la inte- } \\
\text { gración de estudiantes con discapacidad en } \\
\text { la enseñanza universitaria }\end{array}$ & $\begin{array}{l}\text { Con discapa- } \\
\text { cidad }\end{array}$ \\
\hline $\begin{array}{l}\text { Díaz } \\
\text { Voladeras }\end{array}$ & 2009 & $\begin{array}{l}\text { Ir a la universidad. La decisión vocacional de } \\
\text { 1@s adolescentes con discapacidad }\end{array}$ & $\begin{array}{l}\text { Con discapa- } \\
\text { cidad }\end{array}$ \\
\hline Figuera & 2009 & $\begin{array}{l}\text { La transición a la universidad de las perso- } \\
\text { nas con discapacidad }\end{array}$ & $\begin{array}{l}\text { Con discapa- } \\
\text { cidad }\end{array}$ \\
\hline
\end{tabular}




\begin{tabular}{|c|c|c|c|}
\hline Director & Año & Título de la investigación & $\begin{array}{l}\text { Colectivo } \\
\text { considerado }\end{array}$ \\
\hline Gairín & 2009 & $\begin{array}{l}\text { El acceso a la universidad a personas mayo- } \\
\text { res y sin titulación académica }\end{array}$ & Adultos \\
\hline Salmerón & 2009 & $\begin{array}{l}\text { Inclusión de estudiantes magrebíes en las } \\
\text { universidades españolas: desarrollo de com- } \\
\text { petencias personales }\end{array}$ & Inmigrantes \\
\hline Arroyo & 2010 & $\begin{array}{l}\text { Estudio del impacto de los metaversos como } \\
\text { campus espejo para la plena integración de } \\
\text { las personas con discapacidad }\end{array}$ & $\begin{array}{l}\text { Con discapa- } \\
\text { cidad }\end{array}$ \\
\hline Patricio & 2010 & Mediare & $\begin{array}{l}\text { Con discapa- } \\
\text { cidad }\end{array}$ \\
\hline Guasch & 2011 & $\begin{array}{l}\text { Guía de responsabilidad social universitaria } \\
\text { y discapacidad: RSU_D }\end{array}$ & $\begin{array}{l}\text { Con discapa- } \\
\text { cidad }\end{array}$ \\
\hline Michavila & 2011 & $\begin{array}{l}\text { Análisis de las políticas y estrategias de aco- } \\
\text { gida e integración de los estudiantes de nue- } \\
\text { vo ingreso en las universidades españolas }\end{array}$ & Inmigrantes \\
\hline
\end{tabular}

Tabla 2. Estudios financiados con cargo al programa Estudios y Análisis del MEC sobre algunos colectivos de estudiantes en situación de desventaja (2001-2012)

Los informes correspondientes a estos estudios pueden consultarse en: http://138.4.83.162/mec/ayudas/casaAva.asp

El repaso a los resultados de los estudios que figuran en la tabla requiere, obviamente, guardar algunas cautelas. En primer lugar, es necesario apuntar que existe un mayor número de investigaciones destinadas a los estudiantes con discapacidad que a los otros dos colectivos. Además, debe tenerse en cuenta que los trabajos analizados no siempre abarcan las mismas zonas geográficas dentro del territorio español. En algunos casos, adicionalmente, el objetivo general de la investigación es más amplio que el análisis de las medidas dirigidas a los estudiantes con desventaja. No obstante, a partir de estas investigaciones es posible ofrecer algunas ideas preliminares sobre los servicios y apoyos para los estudiantes.

En concreto, una primera consideración que puede plantearse a partir de la revisión realizada es la importancia creciente que la atención a los colectivos de estudiantes con mayores dificultades ha despertado entre la comunidad investigadora. Así, con el paso del tiempo, el número de estudios se ha ido incrementando progresivamente, lo que puede ser un indicio de la mayor preocupación que existe en nuestro país por hacer realidad el principio de equidad en la enseñanza universitaria. 
Por otra parte, si atendemos a los tres colectivos objeto de atención, podemos extraer algunas conclusiones:

a) En relación a los estudiantes de origen inmigrante, algunas universidades han implantado en los años pasados medidas destinadas a facilitar su acceso a los estudios, aunque sólo una minoría de ellas ofrece cursos de pre-acceso diseñados de manera específica para estos alumnos o apoyos económicos para este colectivo. Es más habitual, por el contrario, la existencia de servicios de información y asesoría legal en materia de trámites, homologaciones y convalidaciones. En algunas universidades concretas se han implantado también apoyos psicopedagógicos para estos alumnos. No obstante, y a pesar del notable aumento de la población inmigrante en nuestro país durante los pasados años, los servicios ofertados a los estudiantes de origen inmigrante son todavía escasos en la mayor parte de las universidades.

b) Por lo que se refiere a los estudiantes adultos las investigaciones permiten afirmar también que las iniciativas destinadas de manera concreta a este colectivo son aún escasas en España. En relación con el acceso, aunque se ofertan los sistemas establecidos por la legislación para mayores de 25, 40 o 45 años, son pocas las universidades que cuentan con servicios de orientación o de validación de la experiencia laboral para el acceso de las personas adultas sin titulación. Por otra parte, y a diferencia de lo que sucede en otros países, en la mayoría de las universidades españolas no existen puntos de información para estudiantes adultos, programas de ayuda al estudio o iniciativas para flexibilizar horarios y cursos. La ausencia casi generalizada de servicios de asesoramiento psicopedagógico y de centros de apoyo al aprendizaje dirigidos a estudiantes adultos hace muy difícil para este colectivo obtener el soporte que a menudo necesita. En este sentido, la implantación de cursos o talleres de técnicas de estudio, de estrategias de aprendizaje, de refuerzo académico o de tutoría entre iguales serían iniciativas oportunas.

c) En contraste con los dos colectivos anteriores, los estudios que abordan la atención a los estudiantes con discapacidad indican que la preocupación cada vez mayor que existe por estos alumnos en las universidades se ha traducido en los últimos años en una ampliación de los apoyos y servicios ofrecidos a los mismos, especialmente en forma de ayudas de carácter material. Además, muchas universidades cuentan con programas de prevención, acogida, inclusión en la vida universitaria, extensión universitaria y orientación, así 
como con una oferta formativa extra-académica (deportiva y cultural) adaptada a este tipo de estudiantes. De igual modo, también ha avanzado la oferta de servicios para la transición de estos alumnos al mundo laboral, en muchos casos en colaboración con empresas y otras entidades. No obstante, todavía existen algunos campus que no disponen de espacios adaptados o de recursos de enseñanza apropiados para estos alumnos. En muchos casos se pone de manifiesto también un desconocimiento prácticamente generalizado entre el profesorado acerca de las metodologías pedagógicas más adecuadas para este tipo de alumnos. Adicionalmente es importante destacar que los avances experimentados se concentran básicamente en los apoyos para estudiantes con discapacidades físicas o sensoriales, siendo prácticamente inexistentes para personas con otros tipos de discapacidad.

\section{DISCUSIÓN}

A partir de lo expuesto en las páginas anteriores, pueden extraerse algunas conclusiones relacionadas con la dimensión social del proceso de Bolonia, en términos generales, y con la aplicación de este principio de forma más concreta en las universidades españolas.

Así, es posible afirmar que la dimensión social constituye un eje fundamental del EEES y un concepto básico para dar respuesta a uno de los pilares de las políticas de la Unión Europea, como es la cohesión social. En la base de la dimensión social se encuentran los principios de accesibilidad, igualdad de oportunidades y no discriminación, que pretenden garantizar los derechos del conjunto de los ciudadanos a la educación superior.

De hecho, aunque en los primeros pasos del proceso de convergencia universitaria apenas se prestó atención a la dimensión social, muy poco tiempo después, a iniciativa de los estudiantes, comienza a hablarse de la dimensión social. Las sucesivas reuniones de los ministros responsables de la educación superior de los países participantes en el proceso de Bolonia marcan hitos importantes en el impulso y el desarrollo de esta dimensión, que viene acompañada por la acción de un Grupo de Seguimiento de la misma, en el que los avances se traducen a indicadores de progreso.

En el terreno de la práctica universitaria, los principios que están en la base de la dimensión social se operativizan a través de programas, ayudas y servicios de apoyo a los estudiantes, que atienden a la diversidad de necesidades y de situaciones de los mismos y que contribuyen a impulsar la igualdad de oportunidades. En el caso de España, puede afirmarse que 
dichos servicios han experimentado un incremento progresivo muy importante, incorporándose al organigrama de las universidades y aumentando su oferta en los últimos años. Esta preocupación se refleja también en la investigación sobre la temática, que se ha incrementado de forma notable en los últimos años.

No obstante lo anterior, la revisión de los servicios disponibles en las universidades españolas para tres colectivos concretos de estudiantes, como son los inmigrantes, los estudiantes adultos y los estudiantes con discapacidad, permite concluir que en estos momentos existe una amplia variabilidad entre universidades en los grados de implantación y desarrollo de los servicios disponibles.

Los servicios más extendidos en las universidades españolas son los destinados a estudiantes con discapacidad, en concreto los referidos a estudiantes que presentan discapacidades físicas o sensoriales. Para estos alumnos los apoyos existentes se orientan tanto a facilitar el acceso a la enseñanza universitaria como a ayudarles en el desarrollo de los estudios y en la posterior inserción en el mundo laboral. El progreso experimentado en los últimos años en la atención a este tipo de alumnado ha sido enorme, pero todavía queda mucho por hacer, ya que en algunas universidades aún existen barreras arquitectónicas y escasos recursos para la inclusión de estos estudiantes. Además, es necesario sensibilizar a la comunidad universitaria y formar a los profesores en las metodologías pedagógicas adecuadas para estos alumnos.

Los servicios para estudiantes inmigrantes también se han incrementado en cierta medida en los últimos años en nuestras instituciones de enseñanza superior. Sin embargo, en este caso la tendencia no puede considerarse generalizada, existiendo marcadas diferencias entre universidades. La presencia de la inmigración en nuestro país demandaría una mayor atención a este colectivo, cuyo potencial puede quedar desaprovechado por la falta de apoyos diseñados específicamente para ellos. A este respecto es preciso señalar que los apoyos existentes se centran casi de manera exclusiva en facilitar el acceso a la enseñanza universitaria, sin que existan servicios que puedan ayudar a resolver las necesidades específicas que estos estudiantes puedan tener durante el desarrollo de los estudios o a la hora de ingresar en el mercado laboral. Facilitar el acceso a la universidad es sólo un aspecto inicial de la igualdad de oportunidades, que requiere también medidas destinadas a evitar el fracaso académico de este tipo de alumnos. Este es, por tanto, un ámbito en el que las universidades deberían trabajar a partir de este momento. 
Los servicios dirigidos de manera específica a estudiantes adultos son, en líneas generales, los que han experimentado un incremento menor en nuestro país y siguen estando limitados a ciertas facilidades en el acceso y a apoyos puntuales para quienes tienen responsabilidades familiares. Probablemente se considera que estos estudiantes no precisan de atenciones específicas, pero lo cierto es que la flexibilización de los estudios y los servicios de apoyo para ellos resultan necesarios si aspiramos a equiparar nuestras cifras de estudiantes mayores a las de nuestros vecinos europeos y a abrir oportunidades para que las personas estudien en las distintas etapas de su vida, haciendo del principio de aprendizaje permanente una realidad en nuestra enseñanza superior.

Como conclusión de carácter general se deriva la necesidad de seguir avanzando en la implantación de servicios de apoyo para los estudiantes menos representados en las universidades españolas. Tanto las administraciones competentes como las propias universidades deben potenciar y dedicar los recursos necesarios para hacer efectiva la dimensión social del EEES en nuestro país. Al mismo tiempo es preciso incrementar las investigaciones dedicadas a esta temática, con el fin de conocer de forma detallada las necesidades de los estudiantes con escasa representación y diseñar los apoyos necesarios para los mismos, que, a su vez, deben ser objeto de un seguimiento y una evaluación sistemática para lograr una mejora continua.

\section{NOTAS}

1 Véase http://www.educacion.gob.es/ifiie/investigacion-innovacion/estudios-sistemas-educativos/espanol.html

2 Véase http://www.educacion.gob.es/horizontales/estadisticas/universitaria/alumnado.html 


\section{REFERENCIAS BIBLIOGRÁFICAS}

Alcedo, M. A; Aguado, A. L.; Real, S.; González, M. y Rueda, B. (2007). Una revisión actualizada de la situación de los estudiantes con discapacidad en la Universidad. Anuario de Psicología Clínica y de la Salud (3), 7-18.

Ariño, A. y Llopis, R. (2011a). ¿Universidad sin clases? Condiciones de vida de los estudiantes universitarios en España (Eurostudent IV). Madrid: Ministerio de Educación.

Ariño, A. y Llopis, R. (2011b). Primera encuesta de Condiciones de Vida y Participación de los Estudiantes Universitarios en España (ECoViPEU). Recuperado de: http://www.campusvivendi.com/servicios/ecovipeu/

BFUG - Bologna Process Working Group on Social Dimension and Data on Mobility of Staff and Students in Participating Countries (2007). Key issues for the European Higher Education Area-Social Dimension and Mobility. Stockholm: Government Offices of Sweden. Recuperado de: http://www. ond.vlaanderen.be/hogeronderwijs/ bologna/documents/WGR2007/Socialdimensionandmobilityreport.pdf

Cachón L. (2003). Inmigrantes jóvenes en España. Sistema educativo y mercado de trabajo. Madrid: Injuve.

CERMI (2004). Atención educativa a las personas con discapacidad. Madrid: Comité Español de Representantes de Personas con Discapacidad.

Conferencia de Ministros Europeos Responsables de Educación Superior (2001). Towards the European Higher Education Area. Communique of the meeting of European Ministres in charge or Higher Education en Prague on May 19th 2001. Recuperado de: http://www.crue.org

Conferencia de Ministros Europeos Responsables de Educación Superior (2003). Realising the European Higher Education Area. Communique of the Conference of Ministers responsable for Higher Education in Berlin on 19 September 2003. Recuperado de: http:// www.crue.org/pdf/DeclaracionBerlin2003.pdf

Conferencia de Ministros Europeos Responsables de Educación Superior (2005). The European Higher Education Area - Achieving the Goals. Communiqué of the Conference of European Ministers Responsible for Higher Education. Bergen, 19-20 May. Recuperado de: http://www.crue.org/espaeuro/ lastdocs/Bergen\%20Communique.pdf

Conferencia de Ministros Europeos Responsables de Educación Superior (2007). Towards the European Higher Education Area: Responding to Challenges in a Globalised World. London, 18 May. Recuperado de: http://www.cicdi.ca/docs/ bologna/2007LondonCommunique. en.pdf

Conferencia de Ministros Europeos Responsables de Educación Superior (2009). The Bologna Process 2020 - The European Higher Education Area in the new decade. Leuven, 28-29 Apr. Recuperado de: http://www.aqu.cat/doc/ doc_17546905_1.pdf

Conferencia de Ministros Europeos Responsables de Educación Superior (2012). Making the Most of Our Potential: Consolidating the European Higher Education Area. Bucharest, 26-27 Apr. Recuperado de: http://www.ehea.info/ Uploads/(1)/Bucharest\%20Communique\%202012(2).pdf 
Council of European Union (2010). Council Conclusions of 11 May 2010 on the Social Dimension of Education and Training. Official Journal of the European Union. C 135/2 26.05.2010. Recuperado de: http://eur-lex.europa. eu/LexUriServ/LexUriServ.do?uri=OJ: C:2010:135:0002:0007:EN:PDF

Declaración Conjunta de los Ministros de Francia, Alemania, Italia y el Reino Unido (1998). Declaración conjunta para la armonización del diseño del Sistema de Educación Superior Europeo de 25 de mayo de 1998: Declaración de la Sorbona. Recuperado de: http:// www.eees.ua.es/documentos/declaracion_sorbona.htm

Declaración Conjunta de los Ministros Europeos de Educación (1999). El Espacio Europeo de Enseñanza Superior. Declaración conjunta de los Ministros Europeos de Educación reunidos en Bolonia el 19 de Junio de 1999: Declaración de Bolonia. Recuperado de: http:// www.crue.org

Delors, J. (1996). La educación encierra un tesoro. Informe a la UNESCO de la Comisión Internacional sobre la Educación para el Siglo XXI. Madrid: Santillana-UNESCO.

ESIB - The European Students' Union (2003). European Student Handbook on Equality and Equal Access in Higher Education. Recuperado de: http:// www.esib.org

ESIB - The European Students' Union (2007). Bologna with Student Eyes. Recuperado de: http://www.esib.org

ESU - The European Students' Union (2012). Bologna with Student Eyes. Recuperado de: http://www.esib.org

European Agency for Development in Special Needs Education (2011). Planificación de politicas para implementar la educación inclusiva. Análisis de los retos y oportunidades en la elaboración de indicadores. Recuperado de: http://www.european-agency.org/ agency-projects/mapping-the-implementation-of-policy-for-inclusive-education/agency-flyers

European Association for Quality Assurance in Higher Education (2005). Standards and Guidelines for Quality Assurance in the European Higher Education Area. Helsinki: European Association for Quality Assurance in Higher Education.

European Commission (2001). Lifelong Learning: the implications for universities in the EU. Brussels: European Commission.

European University Association (2005). Glasgow Declaration: Strong Universities for a strong Europe: Brussels: EUA.

Eurostat-Eurostudent (2009). The Bologna Process in Higher Education in Europe. Key Indicators on the Social Dimension and Mobility. Luxembourg: Office for Official Publications of the European Commission.

Eurydice (2011). Modernisation of Higher Education in Europe: Funding and the Social Dimension. Brussels: Education, Audiovisual and Culture Executive Agency.

Fernández Fernández, F. (2008). La vida académica de los estudiantes universitarios españoles en el contexto del Espacio Superior de Educación Europeo. Recuperado de: http://82.223.210.121/ mec/ayudas/repositorio/20080507152 704MEMORIA\%20FINAL\%20INVESTIGACION\%20EA2007URJC-FINALIZADO-A.pdf

Forteza, D. y Ortego, J. L. (2003). Los servicios y programas de apoyo universitarios para personas con discapacidad. Estándares de calidad, acción y evaluación. Revista de Educación Es- 
pecial, 33, 9-26.

Fundación BBVA (2003). Encuesta sobre los estudiantes de enseñanza superior. Bilbao: Departamento de Estudios Sociales y Opinión Pública.

Fundación BBVA (2005). Estudio sobre los universitarios españoles. Bilbao: Departamento de Estudios Sociales y Opinión Pública.

Fundación BBVA (2006). Estudio sobre los universitarios españoles. Bilbao: Departamento de Estudios Sociales y Opinión Pública.

Fundación BBVA (2010). Estudio internacional sobre estudiantes universitarios de seis países europeos. Bilbao: Departamento de Estudios Sociales y Opinión Pública.

González-Badía, J. y Molina, C. (2006). Situación de los estudiantes con discapacidad en las universidades españolas. Fiapas, 111, 30-33.

Hernández Armenteros, J. (coord.) (2002). Información académica, productiva y financiera de las Universidades públicas de España. Madrid: CRUE.

Hernández Armenteros, J. (coord.) (2004). Información académica, productiva y financiera de las Universidades españolas. Indicadores universitarios. Madrid: CRUE.

Hernández Armenteros, J. (coord.) (2006). La universidad española en cifras. Madrid: CRUE.

Hernández Armenteros, J. (coord.) (2008). Información académica, productiva y financiera de las universidades españolas. Indicadores universitarios. Madrid: CRUE.

INJUVE (2008): Informe de la Juventud 2008. Madrid: Instituto de la Juventud.
Lebrero, M. P. y Quicios, M. P. (2010). El estudiante inmigrante y su inclusión en la Universidad española. Educación $X X 1,13(2), 241-262$.

Macmillan, J. y Schumacher, S. (2005). Investigación Educativa. Una introducción conceptual. Madrid: Pearson.

Michavila, F. (dir.) (2012). La Universidad española en cifras. Madrid: CRUE.

Montero, I. y León, O. G. (2007). A guide for naming research studies in Psychology. International Journal of Clinical and Health Psychology, 7, 847-862.

Orr, D., Gwosc, C. y Netz, N. (2011). Social and Economic Conditions of Student Life in Europe. Synopsis of Indicators. Final report Eurostudent IV: 2008-2011. Bielefeld: HIS HochschulInformation-System. Recuperado de: http://www.eurostudent.eu/download_ files/documents/EIV_Synopsis_of_Indicators.pdf

Pena, M. A. y Galindo, A. (eds.) (2005). Inmigración y universidad. Acogida del inmigrante desde el ámbito universitario español. Salamanca: Publicaciones de la Universidad Pontificia de Salamanca.

Real Patronato sobre Discapacidad (2007). Libro Blanco sobre Universidad y discapacidad. Madrid: Ministerio de Trabajo y Asuntos Sociales.

Ramos-Álvarez, M., Moreno-Fernández, M. M., Valdés-Conroy, B. y Catena, A. (2008). Criteria of the peer review process for publication of experimental and quasi-experimental research in Psychology: A guide for creating research papers. International Journal of Clinical and Health Psychology, 8, 751-764.

Ruesga, S. M. (2006). Condiciones sociales y económicas de vida de los estudiantes universitarios en España. 
Proyecto subvencionado por el Ministerio de Educación y Ciencia en el Programa de Estudios y Análisis (Ref. EA 2006-0002). Recuperado de: http:// www.centrorecursos.com/mec/ayudas/ CasaVer.asp?P=29 173

Ruesga, S.M. (2007). Estudiantes universitarios en España: características y competencias en la dimensión Internacional: EUROSTUDENT 2007. Recuperado de: http://82.223.210.121/mec/ ayudas/repositorio/20080530111004E UROSTUDENT-2007.pdf

Ruesga, S.M. y Heredero, M. I. (dirs.) (2008). Los estudiantes universitarios en España: rendimiento académico en relación con el empleo y la movilidad internacional. Recuperado de: http://82.223.160.188/mec/ ayudas/CasaVer.asp?P=29 319
Secretaría General de Universidades (2011): Datos y cifras del sistema universitario español. Curso 20102011. Madrid: Ministerio de Educación.

Skager, R. (1984). Organizing Schools to Encourage Self-Direction in Learners. Hamburg: Pergamon/UNESCO Institute for Education.

Susinos, T. y Rojas, S. (2003). Los servicios de apoyo universitarios y la orientación para el acceso a la universidad. Revista de Educación Especial, 33, 27-38.

Villa, A. y Villa, O. (2007). El aprendizaje basado en competencias y el desarrollo de la dimensión social en las universidades. Educar, 40, 15-48. 


\section{PERFIL ACADÉMICO Y PROFESIONAL DEL/LOS AUTOR/ES}

Inmaculada Egido Gálvez, Doctora en Ciencias de la Educación y Profesora Titular de Teoría e Historia de la Educación en la Universidad Complutense de Madrid. Sus principales líneas de investigación son la Educación Comparada y la Política Educativa Comparada, temas en los que tiene publicados más de una docena de libros en el ámbito nacional e internacional y numerosos artículos en revistas de prestigio. Asimismo, participa asiduamente en actividades de perfeccionamiento del profesorado.

M. ' José Fernández Díaz, Profesora Titular del Departamento de Métodos de Investigación y Diagnóstico en Educación de la Facultad de Educación de la Universidad Complutense de Madrid. Es actualmente Decana de dicha Facultad. Sus principales áreas de especialidad son: calidad y evaluación de las organizaciones educativas, medida y metodología de investigación. Dirige un Grupo de Investigación sobre Calidad y evaluación de instituciones educativas, estando su actividad investigadora y sus publicaciones vinculadas a este campo.

Arturo Galán González, Profesor Titular en el Departamento MIDE de la UNED y premio extraordinario de Doctorado. Ha sido Vicedecano de Investigación entre 2006 y 2011 y Coordinador del Máster en Innovación e Investigación en Educación de esta universidad. Es Secretario General de la Sociedad Española de Pedagogía, Director Adjunto de la Revista Bordón y Vicepresidente de la Asociación para la Investigación y la Docencia Universitas. Research Scholar en Boston University y en el M.I.T. Dirige el Grupo de investigación ESPYD (Educación Superior Presencial y a Distancia).

Dirección de los autores: Inmaculada Egido Gálvez

y M. ${ }^{a}$ José Fernández Díaz

Facultad de Educación de la Universidad

Complutense de Madrid

C/ Rector Royo Villanova s/n

28040 Madrid

E-mail: miegido@edu.ucm.es

E-mail: mjfdiaz@edu.ucm.es

Arturo Galán

Dpto. de Métodos de Investigación

y Diagnóstico en Educación I

Facultad de Educación. Universidad

Nacional de Educación a Distancia (UNED)

C/ Juan del Rosal n. ${ }^{\circ}$ 14. Despacho 219

28040 Madrid

E-mail: agalan@edu.uned.es

Fecha Recepción del Artículo: 25. Enero. 2012

Fecha Modificación del Artículo: 14. Abril. 2012

Fecha Aceptación del Artículo: 19. Abril. 2012

Fecha Revisión para publicación 15. Enero. 2014 